\title{
CEBAF-PR-QZ-O14
}

\section{THE CEBAF PROJECT AND THE FEW-BODY RESEARCH PROGRAM}

\author{
A. Saha \\ Physics Division, CEBAF \\ 12000 Jefferson Avenue, Newport News, VA 23606, USA
}

\begin{abstract}
A major portion of the initial CEBAF physics program in all three experimental halls addresses the study of the nucleon and the few nucleon systems $(A \leq 4)$ using coincidence experiments and emphasizing measurements of polarization observables. The program encompasses the systematic measurement of the elementary form factors of the nucleon to high $\mathrm{Q}^{2}$, the electro- and photo-disintegration of the deuteron and the short distance behaviour of ${ }^{3} \mathrm{He}$ and ${ }^{4} \mathrm{He}$. We present here a brief description of the CEBAF project and the few-body research program planned for Hall A at CEBAF.
\end{abstract}

\section{INTRODUCTION}

Electron scattering reactions have provided a wealth of information about the structure of nucleons and nuclei. Since electrons interact weakly with the nuclear system and since the electromagnetic interaction is well understood, one can deduce quantitative information about the electromagnetic structure of the nucleus under study. With increasing energy and momentum transfers one hopes to observe properties of the nucleus that unambiguously necessitate the modification of the standard meson nucleon description of the nuclear force by a more general description in terms of quarks and gluons in QCD.

In this respect the few nucleon systems provide the best laboratory to observe such phenomena. Since the number of nucleons are small, exact nuclear structure calculations can be performed and compared directly to experimental observations. A major portion of the initial physics program at CEBAF therefore addresses the systematic study of the nucleon and the few nucleon systems $(\mathrm{A} \leq 4)$ in all three experimental halls. By detecting particles emitted from the nucleus in coincidence with the scattered electron one can derive more detailed information of the reaction than in inclusive measurements alone. At CE$\mathrm{BAF}$, due to the $100 \%$ duty factor of the electron accelerator, coincidence measurements and measurements of polarization observables will be emphasized.

\section{CEBAF EXPERIMENTAL EQUIPMENT OVERVIEW}

CEBAF is a $4 \mathrm{GeV}$ continuous beam electron accelerator ${ }^{[1]}$ with very good beam characteristics (emittance $=2 \times 10^{-9} \mathrm{~m}$; energy spread $(4 \sigma)=10^{-4}$ ) that is extendable to higher energies in the future. At present CEBAF is designed to deliver independent beams to three end stations with intensities in the range 0.1 to $200 \mu \mathrm{A}$ and with correlated energies in the range 0.5 to $4 \mathrm{GeV}$. A polarized GaAs photocathode electron source is 
being developed in collaboration with the University of Illinois ${ }^{[2]}$ so as to be able to deliver a polarized electron beam of up to $100 \mu \mathrm{A}$ with a polarization $\geq 49 \%$.

The initial sets of complementary equipment designed for the three experimental end stations $^{[3]}$ are now under construction and are descibed briefly:

Hall A. The initial equipment designed for Hall A consists of a matched pair of identical $4 \mathrm{GeV} / \mathrm{c}$ high resolution spectrometers (Fig. 1, Table 1). These will provide, in conjunction with the high quality CEBAF beam, an opportunity to perform exclusive as well as inclusive electromagnetic experiments with both high precision and accuracy. The QQDQ design includes a large iron-dominated dipole with superconducting racetrack coils and focussing properties provided by slanted pole ends and non-uniform field. The three superconducting quadrupoles are $\cos 2 \theta$-type, current dominated magnets with higher multipole correcting coils. The detector system include vertical drift chambers as position detectors ( $\sim 100 \mu \mathrm{m}$ track resolution) and a focal plane polarimeter in the hadron arm.

Hall B. The CEBAF Large Acceptance Spectrometer (CLAS) to be installed in Hall B (Fig. 2) is a 6-coil superconducting toroidal magnet installed around the beam. The six sectors are filled with drift chambers and particle identification chambers. A photon tagging facility is also being constructed. The CLAS will be best suited for experiments for which broad kinematical coverage and high particle multiplicities are necessary. The luminosity available in the CLAS is $\sim 10^{-34} \mathrm{~cm}^{-2} \mathrm{sec}^{-1}$.

Hall C. The initial equipment in Hall $\mathrm{C}$ consists of a $6 \mathrm{GeV} / \mathrm{c}$ High Momentum Spectrometer (HMS; see Fig. 3) and a Short Orbit Spectrometer (SOS) (see Table 1 for characteristics of both). Both spectrometers have moderate resolution $\left(\sim 10^{-3}\right)$ and may be sufficient for some of the few-body physics program. The high momentum capability of the HMS will allow reaching high $Q^{2}$ values. The SOS can be used in conjunction with the HMS for pion and kaon electroproduction experiments.

Targets and Additional Equipment. The physics program on few nucleon systems calls for the use of extended liquid or high pressure gas cryogenic targets ${ }^{[3]}$ capable of achieving high luminosities up to $\sim 5 \times 10^{-38} \mathrm{~cm}^{-2} \mathrm{sec}^{-1}$. The liquid $\mathrm{H}_{2}$ and $\mathrm{D}_{2}$ targets will operate at 17 atm at $20 \mathrm{~K}$ whereas the ${ }^{3} \mathrm{He}$ and ${ }^{4} \mathrm{He}$ gas targets will operate at $20 \mathrm{~K}$ (ultimately $10 \mathrm{~K}$ ) at 70 atm. The target cells have a physical length of $15 \mathrm{~cm}$ and electron beams of up to $200 \mu \mathrm{A}$ will dissipate nearly $1 \mathrm{KW}$ of power on these targets. Polarized solid state $\mathrm{NH}_{3}$ and $\mathrm{ND}_{3}$ targets are also being developed $[4]$ to achieve nearly $100 \%$ polarization at $0.25 \mathrm{~K}$ in a $5 \mathrm{~T}$ magnetic field. User groups are planning to provide additional pieces of equipment. These may include neutron detectors and polarimeters ${ }^{[5]}$, large solid angle scintillator arrays ${ }^{[6]}$, a high resolution $1.3 \mathrm{GeV} / \mathrm{c}^{\mathrm{M}}$ Multi Purpose Spectrometer (MPS) ${ }^{[7]}$ and dedicated facilities for $\left(e, e^{\prime} K^{+}\right)$and other studies ${ }^{[3]}$.

\section{STRUCTURE OF THE NUCLEON}

The electric $\mathrm{G}_{E}$ and magnetic $\mathrm{G}_{M}$ form factors of the nucleon are of fundamental importance in understanding both the nucleon and nuclear structure. They are an essential ingredient of the electromagnetic response function of nuclei and their $Q^{2}$ dependence is a critical testing ground for QCD based models. 
Table 1. CEBAF Spectrometer Characteristics

\begin{tabular}{|l|c|c|c|}
\hline & $\begin{array}{c}\text { Hall A } \\
\text { HRS (2) }\end{array}$ & $\begin{array}{c}\text { Hall C } \\
\text { HMS }\end{array}$ & $\begin{array}{c}\text { Hall C } \\
\text { SOS }\end{array}$ \\
\hline Configuration & QQDQ & QQQD & QDD \\
\hline Optical Length (m) & 23.4 & 24.8 & 7.4 \\
\hline Momentum Range (GeV/c) & $0.3-4$ & $0.5-6$ & $0.2-1.5$ \\
\hline Momentum Acceptance (\%) & 10 & 10 & 40 \\
\hline Momentum Resolution (FWHM) & $10^{-4}$ & $5 \times 10^{-4}$ & $2 \times 10^{-3}$ \\
\hline Angular Range (deg) & $12.5^{\circ}-165^{\circ}$ & $12.5^{\circ}-90^{\circ}$ & $11.6^{\circ}-168^{\circ}$ \\
\hline Position Accuracy (mr) & 0.1 & 0.5 & 0.5 \\
\hline Ang. Resolution: Horiz (mr) & 0.5 & $0.4(0.8)$ & \\
\hline \multicolumn{1}{|c|}{ FWHM) Vert. (mr) } & 1.0 & $0.9(0.8)$ & \\
\hline Solid Angle (msr) & 7 & 6 & 9 \\
\hline Transverse length acceptance (cm) & 10 & 10 & 10 \\
\hline Transverse position resolution (cm) & 0.1 & $3.4(0.6)$ & \\
\hline
\end{tabular}

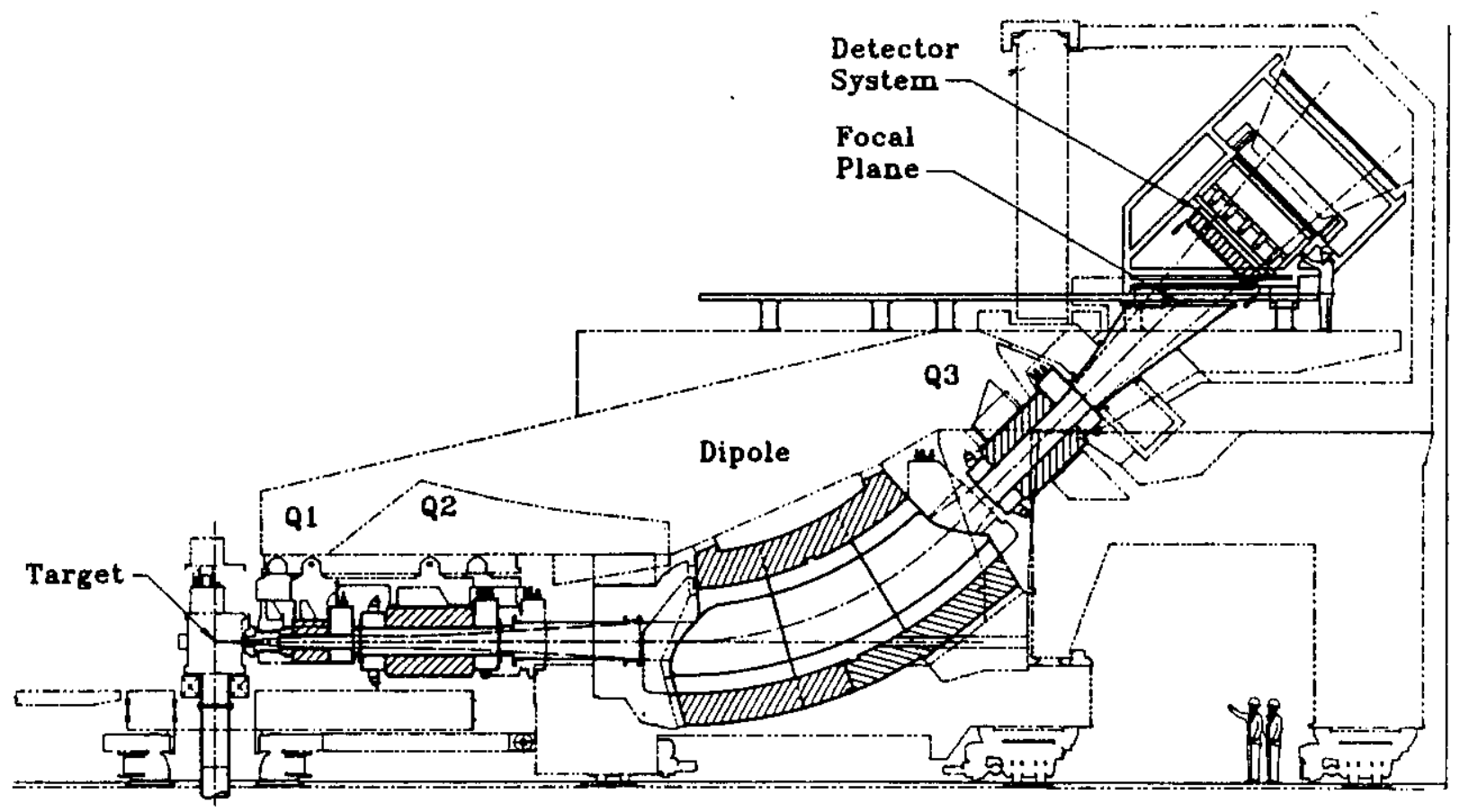

Figure 1. The Hall $A \& \mathrm{GeV} / \mathrm{c}$ High Resolution Spectrometer 


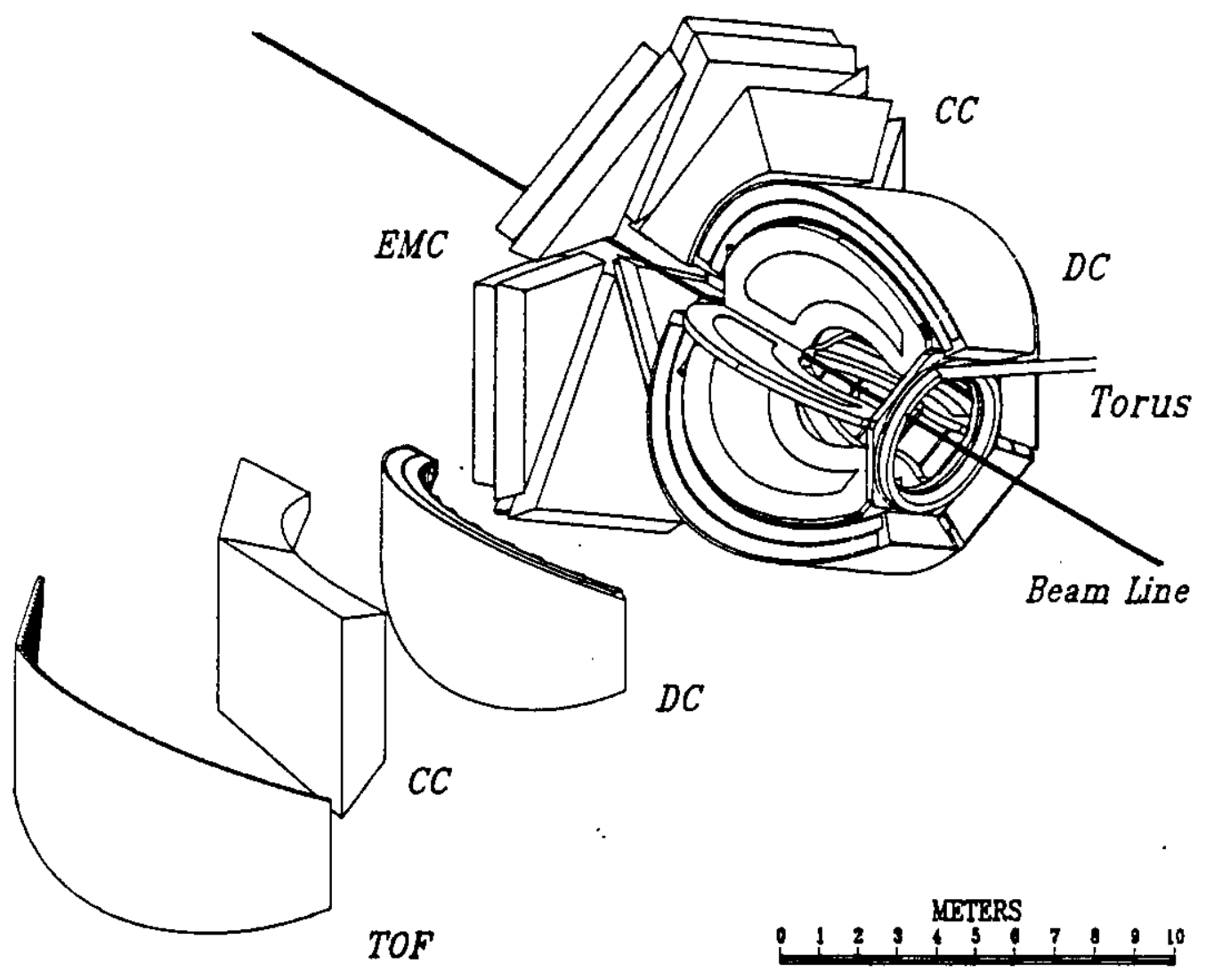

Figure 2. The CEBAF Large Acceptance Spectrometer (CLAS) for Hall B. Drift Chambers (DC), time-of-fight counters (TOF), gas Cerenkov counters (CC), and electrmagnetic calorimeters (EMC) provide particle identification, charged particle tracking and energy measurements for electrmagnetic particles.

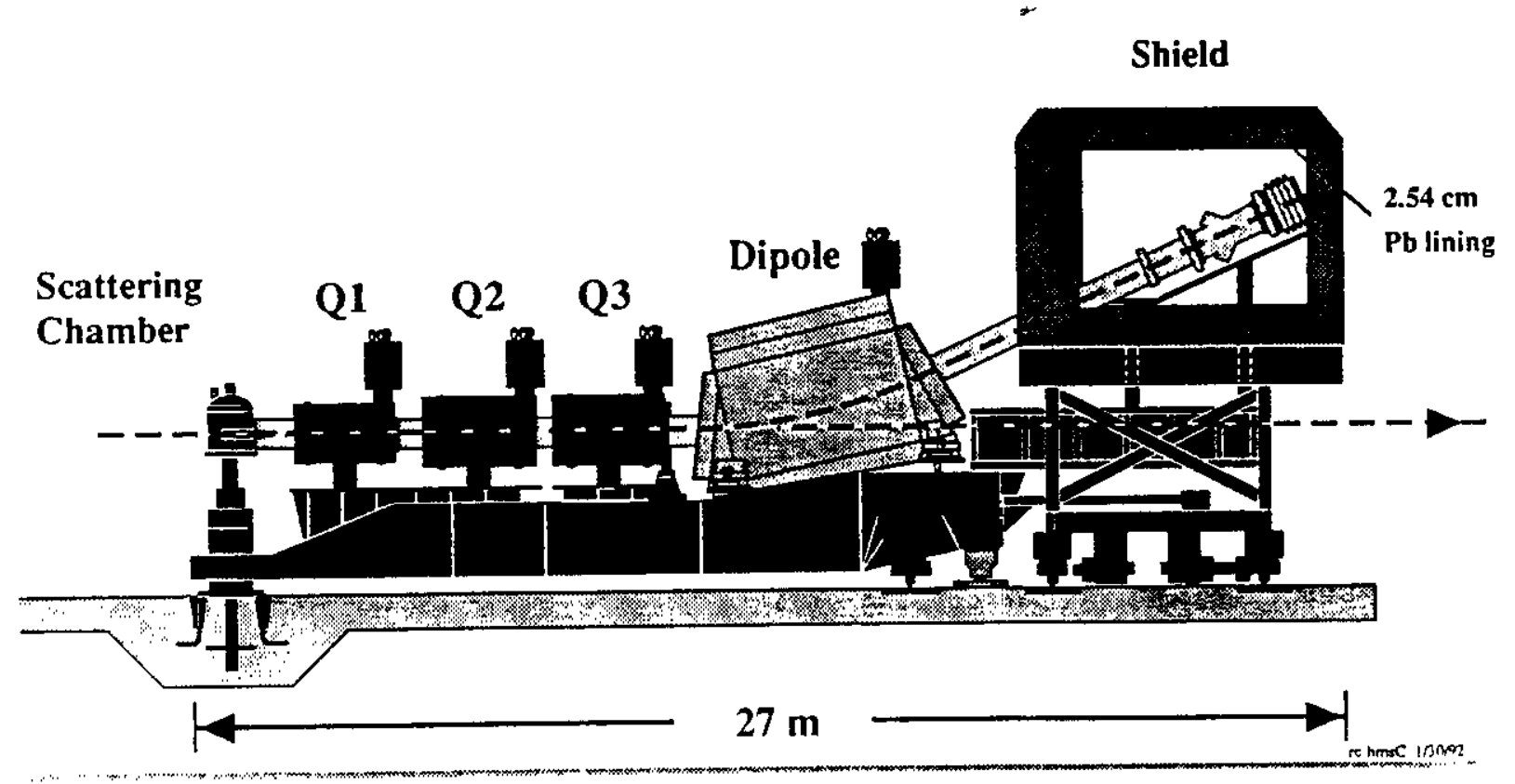

Figure 3. The Hall C High Momentum Spectrometer (HMS). 


\section{(a) Neutron electric form factor $G_{E}^{n}$}

Experimental information on $G_{E}^{n}$ is very poor except for the slope at $Q^{2}=0$. Various models predict different behaviour at large $\mathrm{Q}^{2}$. Experimentally $\mathrm{G}_{E}^{n}$ has been extracted from elastic e-d scattering or inclusive quasielastic e-d scattering using Rosenbluth separation techniques, both of which require the knowledge of the structure of the deuteron in order to correct for coherent effects and the dominant proton contribution. This introduces large uncertainties, as at high $\vec{q}$ the cross section is mainly dominated by $\mathrm{G}_{M}^{P}$ - the transverse component ${ }^{[8,9]}$. Dombey ${ }^{[10]}$ and Arnold, Carlson and Gross ${ }^{[11]}$ have shown that $G_{E}^{n}$ can be determined more accurately if one scatters longitudinaly polarized electrons off a polarized target or measure instead the polarization of the recoiling neutron. Two proposals ${ }^{[12,13]}$ apply these techniques using the coincidence $\left(\vec{e}, e^{\prime} n\right)$ reaction to determine $G_{E}^{n}$. In the first proposal ${ }^{[12]}$, polarized electrons are scattered off solid $\mathrm{ND}_{3}$ target polarized in the scattering plane perpendicular to $\vec{q}$. In the second proposal ${ }^{[13]}$, a recoil neutron polarimeter is used to measure the transverse in-plane polarization of the recoiling neutron. In both cases $G_{E}^{n}$ is extracted from the interference product $G_{E}^{n} G_{M}^{n}$. The uncertainties in $G_{E}^{n}$ obtainable in Proposal [13] for $\mathrm{Q}^{2}$ of up to $1.5(\mathrm{GeV} / \mathrm{c})^{2}$ is shown in Figure 4. Proposal [12] proposes to measure $\mathrm{G}_{E}^{n}$ up to $2(\mathrm{GeV} / \mathrm{c})^{2}$ with nearly similar uncertainties.

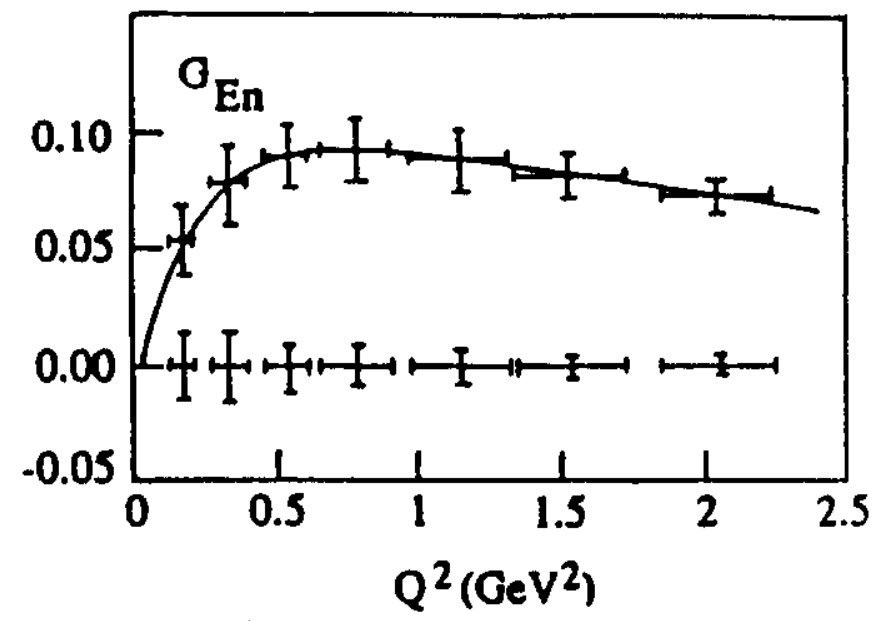

Figure 4. Projected statistical uncertainties in $G_{E}^{n}$ from the proposed experiment ${ }^{2} H\left(\overrightarrow{\mathrm{e}}, e^{\prime} \overrightarrow{\mathbf{n}}\right)^{[13]}$ permit distinguishing between $G_{E}^{n}=0$ and $G_{E}^{n}=-\tau G_{M}^{n}$.

\section{(b) Proton electric form factor $\mathrm{G}_{E}^{\mathrm{p}}$}

Even though the e-p cross section has been measured precisely up to $\mathrm{Q}^{2} \approx 31(\mathrm{GeV} / \mathrm{c})^{2}$, $\mathrm{G}_{E}^{p}$ is poorly known up to only $4(\mathrm{GeV} / \mathrm{c})^{2}$. A recent $\mathrm{SLAC}$ experiment $N E 11^{\{\theta \mid}$ has determined it to $7(\mathrm{GeV} / \mathrm{c})^{2}$ with uncertainties of up to $\pm 15 \%$. The results for $\mathrm{G}_{E}^{p}$ and $\mathrm{G}_{M}^{p}$ are shown in Figure 5. We see that the simple dipole form factor for the proton actually shows the best agreement with the $G_{E}^{P}$ data. As described for the neutron case, $G_{E}^{P}$ can be determined also by performing a coincidence $\left(\vec{e}, e^{\prime} p\right)$ experiment on either a polarized target ${ }^{[12]}$ or measuring the transverse in-plane polarization of the recoiling proton ${ }^{[14]}$. Proposal [14] plans to measure $\mathrm{G}_{E}^{p}$ to $4.5(\mathrm{GeV} / \mathrm{c})^{2}$ with errors of less than $4.6 \%$ using a focal plane polarimeter. Measuring the $\vec{d}\left(\vec{e}, e^{\prime} p\right)$ reaction in quasifree kinematics on a polarized deuteron target ${ }^{[12]}$ or measuring the outgoing recoiling proton ${ }^{[15]}$ provides information on the model for deuteron (which is necessary for the proper extraction of $\mathrm{G}_{E}^{n}$ ) and also gives us a measure of $\mathrm{G}_{E}^{p}$ in the Impulse Approximation where it has been shown $\mathrm{n}^{[16,35,36]}$ that the results are remarkably insensitive to the details of the nuclear model including the choice of wavefunctions, FSI and MEC. 


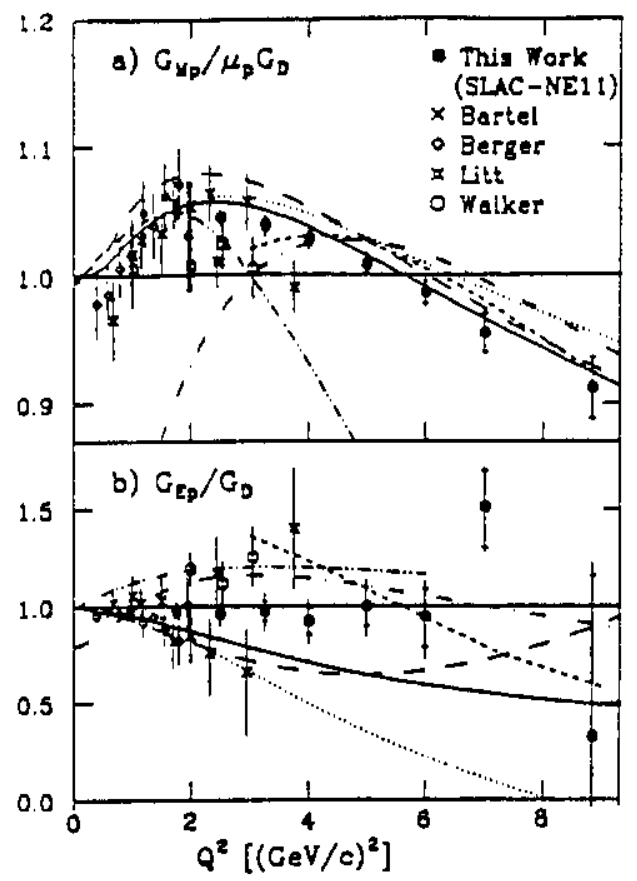

Figure 5. Results from the $S L A C$ experiment $N E 11^{[9]}$ for (a) $G_{M}^{p} / \mu G_{D}(b) G_{E}^{p} / G_{D}$. The inner errors are statistical only, while the outer errors include point-to-point systematic errors. Data from previous experiments and several theoretical fits and predictions are also shown - see reference 9 for details.

\section{(c) The Nucleon Weak Form Factors}

In the Standard Model, the coupling of the neutral weak boson $\mathrm{Z}^{0}$, to the quarks are known ${ }^{[17]}$ and the weak form factors can be expressed in terms of the electromagnetic ones,

$$
\begin{aligned}
& G_{E}^{z}\left(Q^{2}\right)=(1 / 4)\left[\left(1-4 \sin ^{2} \theta_{W}\right) G_{E_{p}}^{\gamma}\left(Q^{2}\right)-G_{E n}^{\gamma}\left(Q^{2}\right)-G_{E}^{s}\left(Q^{2}\right)\right] \\
& G_{M}^{z}\left(Q^{2}\right)=(1 / 4)\left[\left(1-4 \sin ^{2} \theta_{W}\right) G_{M_{p}}^{\gamma}\left(Q^{2}\right)-G_{M_{n}}^{\gamma}\left(Q^{2}\right)-G_{M}^{s}\left(Q^{2}\right)\right]
\end{aligned}
$$

where $G_{E, M}^{s}\left(Q^{2}\right)$ arise from the presence of strange quark-antiquark pairs in the nucleon. Recently, considerable interest in these form factors has been focussed on the possibility that the strange quarks contribute significantly to the nucleon matrix elements. Deep inelastic muon scattering ${ }^{[18]}$ and elastic neutrino scattering ${ }^{[19]}$ have shown surprising results which indicate that strange quarks may have sizeable matrix elements $\left\langle N\left|\vec{s} \gamma_{\mu} \gamma_{5} s\right| N>\right.$ in nucleons and contribute significantly to the form factors. Determination of $G_{E}^{s}$ gives information about the "strangeness radius" of the proton ${ }^{[20,21]}$, whereas determination of $G_{M}^{s}\left(Q^{2}=0\right)$ measures the strange magnetic moment $\mu_{s}$. From simple arguments using vector dominance, these contributions should be negligible ${ }^{[22]}$, whereas phenomenological models using dispersion relations ${ }^{[21]}$ predict $\mu_{s}=-0.31 \pm 0.09$ and the "strangeness radius" $r_{s}^{2}=0.16 \pm 0.06 \mathrm{fm}^{2}$.

One way to measure these new form factors is to determine the parity violating asymmetry in elastic $\overrightarrow{e p}$ scattering.

$$
\begin{aligned}
& A \equiv \frac{\sigma^{\dagger}-\sigma^{\downarrow}}{\sigma^{\top}+\sigma^{\downarrow}} \sim \frac{A_{W} A_{e m}}{\left|A_{e m}\right|^{2}} \\
& =\frac{G_{F} Q^{2}}{\pi \alpha \sqrt{2} \xi}\left[\epsilon G_{E}^{\gamma} G_{E}^{z}+\tau G_{M}^{\gamma} G_{M}^{z}-\frac{1}{2}\left(1-4 \sin ^{2} \theta_{W}\right) \sqrt{1-\epsilon^{2}} \sqrt{\tau(1+\tau)} G_{M}^{\gamma} G_{A}^{z}\right] \\
& \text { where } \xi=\epsilon\left(G_{E_{p}}^{\gamma}\right)^{2}+\tau\left(G_{M_{p}}^{\gamma}\right)^{2}, \epsilon=\left[1+\left(2 q^{2} / Q^{2}\right) \tan ^{2} \theta / 2\right]^{-1} \text { and } \tau=Q^{2} / 4 M^{2}
\end{aligned}
$$


At fixed $Q^{2}$, the role of each of the weak form factors changes as a function of the scattering angle. Separating the form factors requires measurements at three different angles for the same $Q^{2}$. At CEBAF, several proposals ${ }^{[23,24,25]}$ concentrates on determining $G_{E}^{s}$ by measuring the asymmetry at forward angles. In Hall A, parity measurements are made with elastic $\overrightarrow{e p}^{[23]}$ scattering in the $Q^{2}$ range of 0.27 to $1.3(\mathrm{GeV} / \mathrm{c})^{2}$ and with elastic $e^{-4} \mathrm{He}$ scattering in the $\mathrm{Q}^{2}$ range of 0.01 to $0.18(\mathrm{GeV} / \mathrm{c})^{2[23]}$ and at $\mathrm{Q}^{2}=0.6(\mathrm{GeV} / \mathrm{c})^{2[24]}$. Both proposals use the two high resolution spectrometers at forward angles $\left(12.5^{\circ}\right)$. In Hall C, a dedicated 8-coil toroidal spectrometer is proposed ${ }^{[25]}$ to measured the recoil proton in the elastic $\overrightarrow{e p}$ scattering at $Q^{2}$ range 0.1 to $0.3(\mathrm{GeV} / \mathrm{c})^{2}$. An experiment at MIT Bates (SAMPLE experiment) ${ }^{[26]}$ is planning to determine $G_{M}^{s}$ at $\mathrm{Q}^{2} \sim 0.1(\mathrm{GeV} / \mathrm{c})^{2}$ by measuring the elastic $\vec{e}$ p scattering asymmetry at backward angles.

\section{(d) Electroexcitation of Nucleon Resonances}

Electro- and photo- excitation of nucleon resonances gives essential information in the understanding of the structure of hadrons in terms of the fundamental interaction of the constituent quarks and gluons. This information is experimentally accessible through the determination of multipolar components of the transition of the proton to its excited resonant states. Coincident detection of the resonance decay products allow the separation of overlapping resonances and spin/isospin assigninents. These studies will be a major program for the CLAS detector in Hall $\mathrm{B}^{[27]}$. Additional information is obtained by measuring the polarization observables of the recoiling proton in the $\mathrm{p}\left(\vec{e}, \mathrm{e}^{\prime} \overrightarrow{\mathrm{p}}\right) \pi^{0}$ where one is able to access many of the amplitudes that do not occur in unpolarized experiments, which probe only the real parts of the interference amplitudes, or require out-of-plane measurements for their determination. Polarization of the electron beam, the recoil proton, or both are necessary to probe the imaginary parts. A proposal in Hall $\mathrm{A}^{[28]}$ therefore plans to make precise measurements of the $\mathrm{p}\left(\overrightarrow{\mathrm{e}}, \mathrm{e}^{\mathrm{e}} \overrightarrow{\mathrm{p}}\right) \pi^{0}$ reaction at the $\Delta$ and Roper resonances in Hall $\mathrm{A}$ at $\mathrm{Q}^{2}=0.5,1$ and $1.5(\mathrm{GeV} / \mathrm{c})^{2}$ for the $\Delta$ and $\mathrm{Q}^{2}=0.23$ and $0.68(\mathrm{GeV} / \mathrm{c})^{2}$ for the Roper resonance. Six response functions $R_{L T}^{\prime l}, R_{L T}^{\prime t}, R_{L T}^{n}, R_{L T}, R_{T T}^{\prime \prime}$ and $R_{T T}^{\prime t}$ will be separated. In addition, the combinations $2 \epsilon R_{L}^{n}+R_{T}^{n}-\epsilon R_{T T}^{n}$ and $2 \epsilon R_{L}+R_{T}-\epsilon R_{T T}$ will be determined. These measurements will therefore complement the more extensive measurements planned for the CLAS detector.

In the $S U(6)$ spherically symmetric quark model the transition between the proton and the $\Delta^{+}(1232)$ is explained by a single quark spin-flip in the overall S-wave (1s) ${ }^{3}$ ground state corresponding to a magnetic dipole transition $\mathrm{M}_{1+}$. In more realistic QCD based models which include color magnetic interactions the $\Delta$ acquires an $\mathrm{L}=2$ component which leads to a small electric $\left(\mathrm{E}_{1+}\right)$ and scalar $\left(\mathrm{S}_{1+}\right)$ contributions. At very large $\mathrm{Q}^{2}, \mathrm{PQCD}$ predicts $E_{1+} / M_{1+} \rightarrow 1$ and $S_{1+} / M_{1+}$ remains small. Proposal [28] will provide information about $\operatorname{Im}\left(\mathrm{E}_{1+} \mathrm{M}_{1+}^{*}\right)$ and $\operatorname{Im}\left(\mathrm{S}_{1+} \mathrm{M}_{1+}^{*}\right)$ and other polarization response functions which will provide further constraints on various models.

In the simple symmetric quark model, the $\mathrm{P}_{11}(1440)$ Roper resonance is characterized as a "breathing mode" or a radial excitation of one quark to a 2 s state $\left((1 s)^{3} \rightarrow(1 s)^{2}(2 s)\right)$ giving rise to a large longitudinal component along with the magnetic dipole $\left(\mathrm{M}_{1+}\right) \mathrm{com}$ ponent. The nature of the Roper is still in question as it remains uncertain in electroproduction data. The polarization observables for the Roper in Proposal [28] will therefore be able to distinguish between various possibilities: no Roper at all, a Roper with zer" longitudinal coupling as predicted if the Roper is a hybrid ( 3 quarks +1 gluon) baryon 
and more conventional quark models where the Roper is a radial excitation and may have a large longitudinal coupling.

\section{STRUCTURE OF THE DEUTERON}

\section{(a) Deuteron Elastic Form Factors}

Electron scattering from the deuteron has been an extremely important means of understanding the properties of the nuclear two body system. The deuteron elastic form factors $A\left(Q^{2}\right)$ and $B\left(Q^{2}\right)$ offer unique opportunities to test models of the short-range nucleon-nucleon interaction, meson-exchange currents (MEC) and isobaric configurations (IC) as well as possible influence of explicit quark degrees of freedom. Proposal [29] plans to measure forward and $180^{\circ}$ elastic electron-deuteron scattering to the highest momentum transfers possible, limited by a cross section sensitivity of about $2 \times 10^{-42} \mathrm{~cm}^{2} / \mathrm{sr}$ in Hall $A$. The forward angle experiment will extend the knowledge of the electric elastic structure function $A\left(Q^{2}\right)$ up to possibly $Q^{2}=6(\mathrm{GeV} / \mathrm{c})^{2}$ and significantly improve the existing low $\mathrm{Q}^{2}$ data. The backward angle experiment will extend the measurements of the magnetic elastic structure function $\mathrm{B}\left(\mathrm{Q}^{2}\right)$ of the deuteron up to possibly $\mathrm{Q}^{2}=5(\mathrm{GeV} / \mathrm{c})^{2}$ and accurately map its diffraction minimum.

\section{(b) Deuteron Threshold Electrodisintegration}

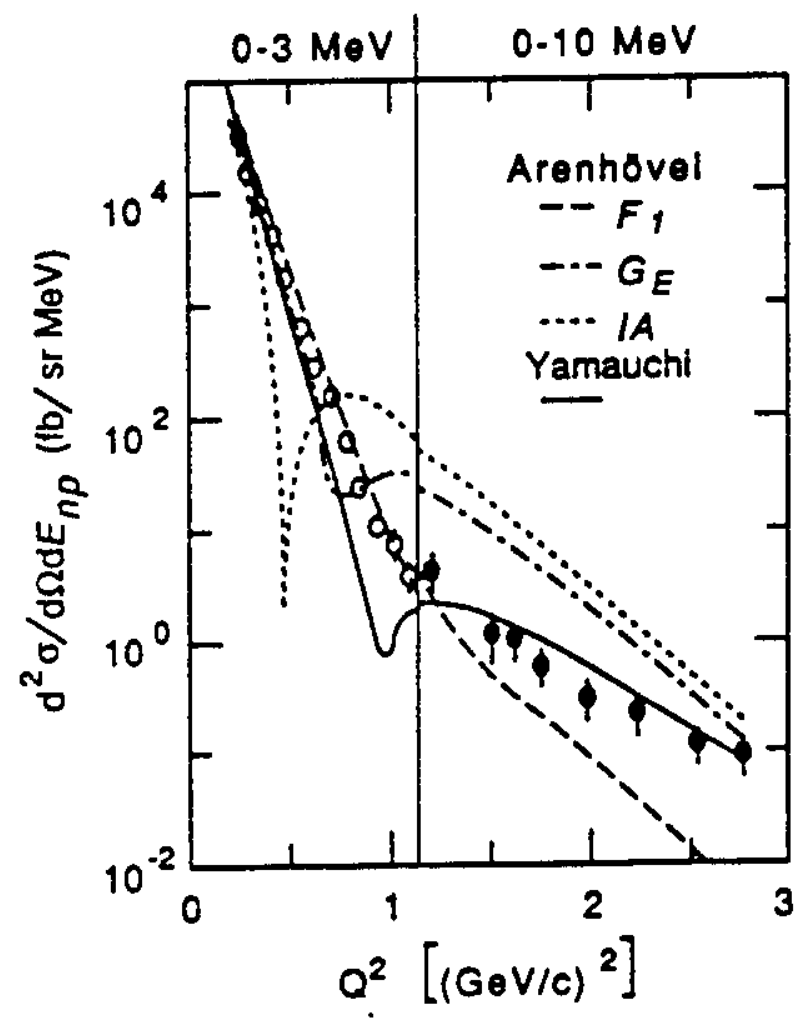

Figure 6. Threshold electrodisintegration cross sections at $180^{\circ}$ vs $Q^{2}$. All predictions and $S L A C$ data $^{[31]}$ above $Q^{2}=1.1(\mathrm{GeV} / \mathrm{c})^{2}$ are averaged over a $10 \mathrm{MeV}$ excitation energy range. Below $Q^{2}=1.1(\mathrm{GeV} / \mathrm{c})^{2}$, the Saclay data ${ }^{[30]}$ (open circles) and Ahrenhovel's meson-nucleon predictions are averaged over $0.3 \mathrm{MeV}$, while the hybrid quark-hadron model of Yamauchi is at $E_{n p}=1.5 \mathrm{MeV}$.

Threshold electrodisintegration of the deuteron $d\left(e, e^{\prime}\right)$ np has provided one of the most direct evidence of non-nucleonic degrees of freedom ${ }^{[30]}$. A recent SLAC measurement $\left(\mathrm{NE}_{4}\right)^{[31]}$ has extended the SACLAY results to $\mathrm{Q}^{2} \approx 2.8(\mathrm{GeV} / \mathrm{c})^{2}$ (see figure 6 ) although with a lower resolution which necessitated averaging over $10 \mathrm{MeV}$, thus precluding any 
separation from the elastic scattering process. The proposal [32] plans to measure the threshold deuteron disintegration with resolution comparable to that obtained at SACLAY $(\approx 1 \mathrm{MeV})$ and with a cross section sensitivity limit of about $5 \times 10^{-42} \mathrm{~cm}^{2} / \mathrm{sr}$. The experiment will provide a measurement of the ${ }^{3} S_{1}+{ }^{3} D_{1} \rightarrow{ }^{1} S_{0}$ transition and the nearby threshold region of higher excitations. The results will place severe constraints on the diverging theoretical predictions at large monentum transfers and will lead to a better understanding of non-nucleonic and quark-gluon degrees of freedom in the deuteron.

\section{(c) Photodisintegration of the Deuteron}

The $\mathrm{d}(\gamma, \mathrm{p}) \mathrm{n}$ reaction is a simple test case for nuclear theories. As the incident $\gamma$ energy is increased, new degrees of freedom become important and can be investigated. Recently, it has been suggested ${ }^{[3]}$ that dimensional scaling has been observed at photon energies above $1.4 \mathrm{GeV}$, which indicates that the reaction is sensitive to the quark substructure of the nucleons. Proposal [34] plans to measure angular distributions of outgoing polarized protons for incident energies from 0.5 to $1.8 \mathrm{GeV}$ for this reaction. This will provide a more sensitive test as spin measurements are typically more sensitive to interference of reaction mechanisms than differential cross section measurements. The results of this experiment will therefore provide a test of the capabilities of conventional nuclear theories and also a sensitive check on the existence of various exotic phenomena such as dibaryons. and the onset of dimensional scaling.

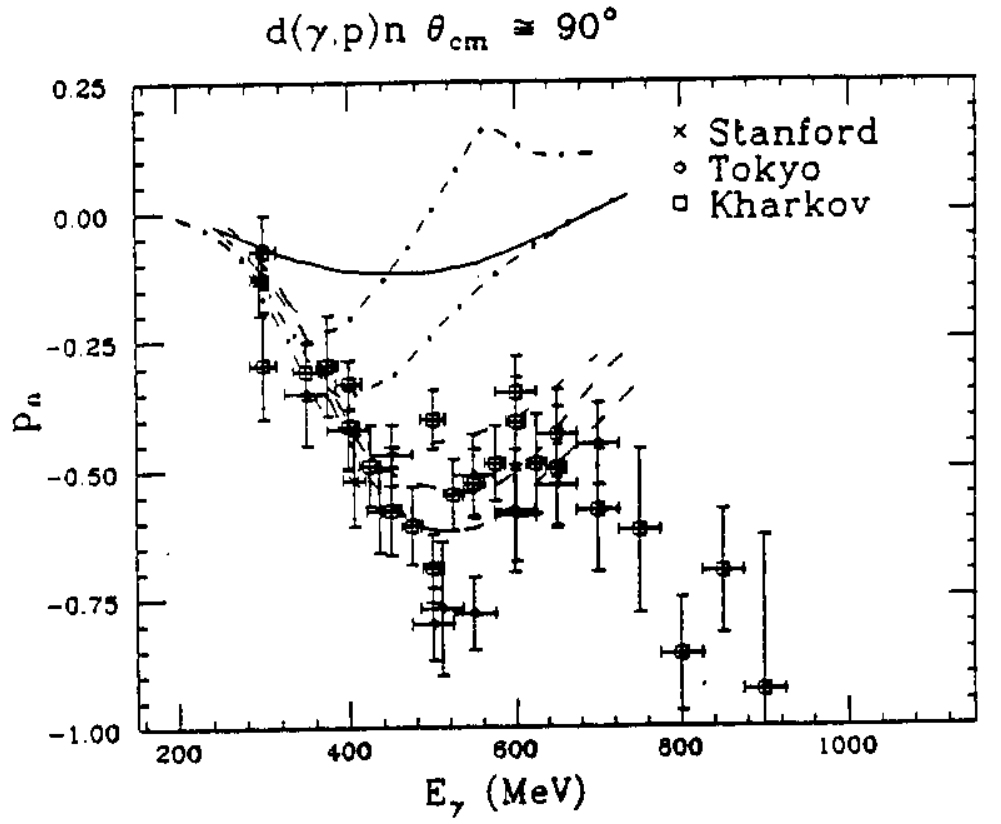

Figure 7. Outgoing proton polar. ization measurements in the $d(\gamma, \vec{p}) n$ reaction at $\theta_{\mathrm{cm}}=90^{\circ}$. The data are from references $[35,36,37] . A$ conventional calculation (solid curve) and conventional + dibaryon calculations (dashed curves) in two different models for the dibaryon ${ }^{[38]}$. The dash-dot curve are from Laget ${ }^{39 \mid}$ and show the sensitivity of his reaction mechanism to the choice of wave function.

\section{(d) Coincidence $\mathrm{D}\left(\mathrm{e}, \mathrm{e}^{\prime} \mathrm{p}\right) \mathrm{n}$ experiments}

Systematic studies of the $d\left(e, e^{\prime} p\right) n$ reaction will be performed in Hall $A$ with high precision and accuracy ${ }^{[15]}$. The $\mathrm{Q}^{2}$ dependence of the reaction will be examined in quasielastic kinematics by performing a longitudinal/transverse separation at $P_{R}=0$ for $Q^{2}$ values in the range 0.23 to $3.4(\mathrm{GeV} / \mathrm{c})^{2}$. The cross sections in the forward and backward direction: will be measured to $1 \%$ accuracy. Moreover an angular distribution of the proton will $b$. 
measured up to $\mathrm{p}_{R}=500 \mathrm{MeV} / \mathrm{c}$ at $\vec{q}=1.0 \mathrm{GeV} / \mathrm{c}$. In these non-parallel kinematics, in addition to the two response functions $R_{L}$ and $R_{T}$, two interference response functions $\mathrm{R}_{L T}$ and $\mathrm{R}_{T T}$ are also present. From in-plane measurements on either side of $\vec{q}$ plus a backward angle measurement, $R_{T}, R_{L T}$ and $R_{L}+R_{T T}$ can be determined and will provide added constraints on the various microscopic models. In Fig. 8 the asymmetry, which is related to the interference response function $\left(R_{L T}\right)$, relative to $\vec{q}$ is shown vs. $\theta_{c m}^{p n}$. Measurements above $\theta_{c m}^{p n} \sim 30^{\circ}$ will provide a sensitive test of FSI models $[16,40]$.

Asymmetry

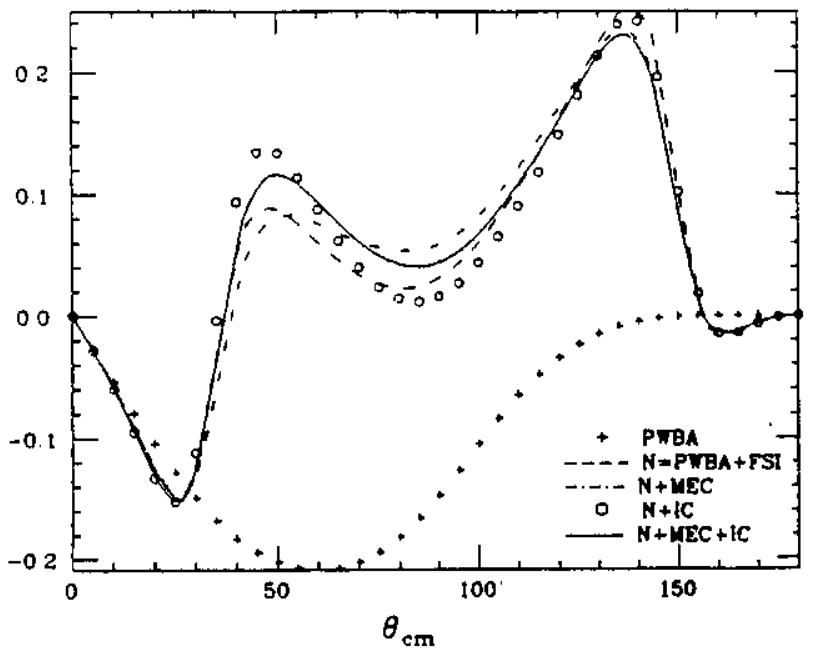

Figure 8. The asymmetry $A=\operatorname{lo}\left(\phi_{x}=\right.$ $\left.0)-\sigma\left(\phi_{x}=\pi\right)\right] /\left[\sigma\left(\phi_{x}=0\right)+\sigma\left(\phi_{x}=\pi\right) / r e l\right.$. ative to $\vec{q}$ as deduced from the four response functions from reference $[16,40]$.

In addition, polarization measurements provide additional tests of nuclear wavefunctions and currents and are sensitive to many features of the reaction mechanism. Fabian and Ahrenhovel $[16,40]$ have made non-relativistic calculations on the deuteron and have shown that there is large sensitivity (50-100\%) to the non-nucleonic degrees of freedom (MEC and IC) along with FSI, especially in the polarization observables. These effects are much weaker in the usual (unpolarized) $R_{L}$ and $R_{T}$ responses. Similar conclusions have been reached by Korchin et al..$^{[41]}$. At higher energies, relativistic calculations have been performed by Rekalo et al. ${ }^{[42]}$ and are underway by Gross and Van Orden ${ }^{[43]}$. The proposal [15] will attempt to measure the recoil proton polarizations in ${ }^{2} \mathrm{H}\left(\overrightarrow{\mathrm{e}}, \mathrm{e}^{\prime} \overrightarrow{\mathrm{p}}\right)$ over a broad range of kinematics. In the first part all three polarization observables will be extracted in perpendicular kinematics at $\vec{q}=1.26 \mathrm{GeV} / \mathrm{c}$ reaching a recoil momentum of $300 \mathrm{MeV} / \mathrm{c}$. The polarization asymmetries about $\vec{q}$ will also be extracted. In the next part, a $\mathrm{Q}^{2} \mathrm{de}$ pendence $\left(0.23\right.$ to $\left.3.24(\mathrm{GeV} / \mathrm{c})^{2}\right)$ of the reaction will be studied at zero recoil mementum. In analogy with the ${ }^{2} \mathrm{~B}\left(\overrightarrow{\mathrm{e}}, e^{\prime} \overrightarrow{\mathrm{n}}\right)$ reaction to measure $G_{\vec{E}}^{n}$ (assuming nuclear correlations can be handled), the ${ }^{2} \mathrm{H}\left(\overrightarrow{\mathrm{e}}, \mathrm{e}^{\prime} \overrightarrow{\mathrm{p}}\right)$ reaction, in conjunction with the $\mathrm{p}\left(\overrightarrow{\mathrm{e}}, \mathrm{e}^{\prime} \overrightarrow{\mathrm{p}}\right)$ reaction to measure $\mathrm{G}_{E}^{p}$, will test the validity of reaction models for the deuteron.

\section{STRUCTURE OF ${ }^{3} \mathrm{He}$ AND ${ }^{4} \mathrm{He}$}

\section{(a) Elastic Form Factors}

The form factors of ${ }^{3} \mathrm{He},{ }^{3} \mathrm{H}$ and ${ }^{4} \mathrm{He}$ have been thoroughly investigated both experimentally and theoretically. Recent measurements ${ }^{[4,45,46]}$ have separated the charge and magnetic form-factors of both trinucleon systems up to $\sim 30 \mathrm{fm}^{-2}$. The charge form factor of ${ }^{4} \mathrm{He}$ has also been measured ${ }^{[44]}$ up to $\mathrm{Q}^{2}=45 \mathrm{fm}^{-2}$. It is evident that the impulse 
approximation (IA) alone cannot describe the data correctly and, as in the case of the deuteron, explicit introduction of non-nurleonic degrees of freedom is necessary. The inclusion of MEC and IC as well as three-body forces provides a reasonable description of the data. Data at higher momentum transfers will determine if explicit sub-nucleonic degrees of freedom are also necessary. Proposal [47] will attempt to measure these form-factors to the highest momentum transfer possible at CEBAF, limited only by a cross section sensitivity of $\sim 2 \times 10^{-42} \mathrm{~cm}^{2} / \mathrm{sr} / \mathrm{MeV}$, thereby possibly extending the existing $\mathrm{Q}^{2}$ range in both ${ }^{3} \mathrm{He}$ and ${ }^{4} \mathrm{He}$ by a factor of two.

\section{(b) High Momentum Components and separation of response functions}

The study of high momentum components in nuclei is of special interest in nuclear physics as it is sensitive to correlations arising from the short range part of the NN interaction. Self-consistent mean field descriptions of nuclei fail to describe these components and significant non-nucleonic degrees of freedom (MEC, IC) are necessary to adequately explain the existing data for $\mathrm{D}^{[48,49]}$ and ${ }^{3} \mathrm{He}^{[50]}$ at high values of recoiling momenta $\left(\mathrm{p}_{R} \approx\right.$ $500 \mathrm{MeV} / \mathrm{c}$ ). These studies have been limited not only to very low momentum transfers $\left(\mathrm{Q}^{2} \sim 0.05(\mathrm{GeV} / \mathrm{c})^{2}\right)$ but were forced, due to the limitations of the presently available facilities, to small values of Bjorken $x(\sim 0.1)$ i.e. well in the dip and delta regions. For heavier nuclei because of the above mentioned experimental limitations, momentum distributions have been restricted to only $\mathrm{p}_{R} \leq 300 \mathrm{MeV} / \mathrm{c}$ and small $\mathrm{x}$. The present proposals plan to measure the momentum distributions in ${ }^{3,4} \mathrm{He}^{[51]}$ (as in $\mathrm{D}^{[15]}$ ) in perpendicular kinematics (where $e_{c m}$ of the final system is kept constant) in the quasielastic region $(x \approx 1)$ up to $p_{R}$ $=500 \mathrm{MeV} / \mathrm{c}$ with momentum transfers $\mathrm{Q}^{2} \approx 0.56(\mathrm{GeV} / \mathrm{c})^{2}$. Plans are to extend these measurements to higher values of $\mathrm{p}_{R}(\sim 800 \mathrm{MeV} / \mathrm{c})$ and $\mathrm{Q}^{2}\left(\sim 1.5(\mathrm{GeV} / \mathrm{c})^{2}\right.$. In these nonparallel kinematics, in addition to the two response functions $R_{L}$ and $R_{T}$, two interference response functions $\mathrm{R}_{L T}$ and $\mathrm{R}_{T T}$ are also present. From in-plane measurements on either side of $\vec{q}$ plus a backward angle measurement, $R_{T}, R_{L T}$ and $R_{L}+R_{T T}$ can be determined and will provide added constraints on the various microscopic models.

(c) Electroexcitation of correlated nucleon pairs in the continuum and short range correlations

The most direct access to two-nucleon density distributions is through two nucleon emission reactions. Information on this can also be obtained from the less exclusive (e, $\left.\mathrm{e}^{\prime} \mathrm{N}\right)$ reaction under kinematics which favour the absorption of the photon on a nucleon pair. Calculations by Ciofi degli Atti et a $f^{[52]}$ in ${ }^{3} \mathrm{He}$ show a definite relationship between highmomentum components and continuum strength. The proton momentum distributions obtained by integrating the one-body spectral function $S\left(\vec{p}, E_{m}\right)$ show that the high momentum strength is completely dominated by correlations and that it is spread over a large continuum in missing energy. This has been clearly demonstrated in ${ }^{3} \mathrm{He}\left(e, e^{\prime} p\right) n p^{[50]}$ and ${ }^{4} \mathrm{He}\left(e, e^{\prime} p\right) n p^{[53]}$. The broad structure observed in both nuclei has the kinematic signature expected from an interaction with a two-nucleon pair, moving to higher values of $E_{m}$ as the recoil momentum $P_{R}$ is increased. Excess of strength at high missing energy in ${ }^{12} \mathrm{C}\left(\mathrm{e}, \mathrm{e}^{\prime} \mathrm{p}\right)$ experiments at Bates in the quasielastic ${ }^{[54]}, \mathrm{dip}^{[55]}$ and quasifree delta region ${ }^{[56]}$ also indicate important contributions from two-nucleon processes. Proposal [51] will vastly extend the kinematic range of these studies on ${ }^{3,4} \mathrm{He}$ and will moreover make an in-plane separation of the response functions in perpendicular kinematics at $Q^{2}=1.0(\mathrm{GeV} / \mathrm{c})^{2}$ and recoil momenta up to $1 \mathrm{GeV} / \mathrm{c}$. 


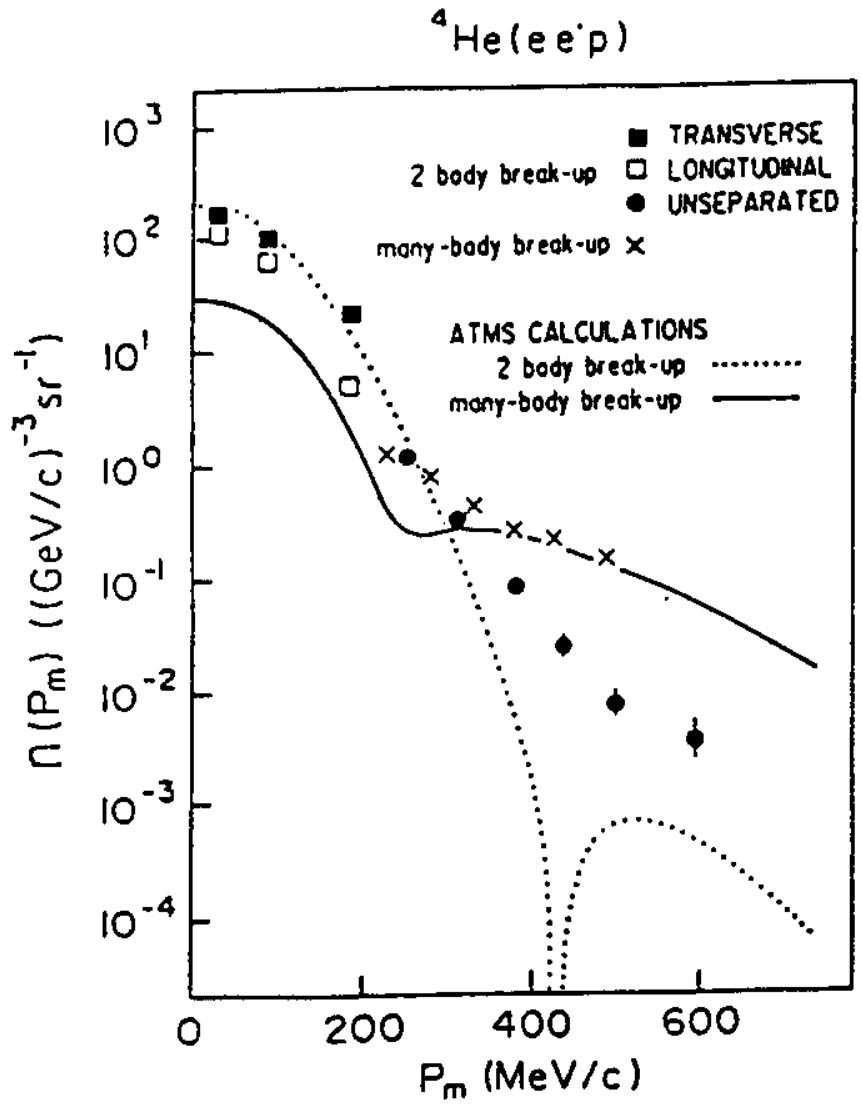

Figure 9. Proton momentum distributions in ${ }^{4} \mathrm{He}$ from the $\left(e, e^{\prime} p\right)$ reaction ${ }^{[53]}$ for the two body break-up, $S\left(19.8 \mathrm{Mev}, \mathrm{p}_{m}\right)$, and for the continuum integrated over the miss. ing energy spectrum: $S\left(e_{m}, P_{m}\right) d e_{m}$. We see that the momentum distribution is dominated by many-body break-up above $300 \mathrm{MeV} / \mathrm{c}$ where short range correlations are present.

As stated earlier, a full investigation of two-nucleon currents and correlations in nuclei requires $\left(e, e^{\prime} 2 \mathrm{~N}\right)$ triple coincidence experiments. Proposal [57] plans to measure e-p-p and $e-p-n$ angular correlation cross sections in ${ }^{3,4} \mathrm{He}$ in geometries which permit simplified interpretation of the data and selectively emphasize various components of the knockout mechanism. Such experiments will provide important and fundamental information on the elusive short range two nucleon correlations, two-body meson exchange currents and associated final state interactions between the ejected nucleons and the nucleus. Proposal [58] offers an efficient way to study two nucleon correlations in nuclei by performing the (e,e'd) reaction instead. By projecting these correlations onto a deuteron in the final state some details are lost since effectively an integral is taken over certain correlations. On the other hand the $\left(e, e^{\prime} d\right)$ reaction can be used as an isospin filter and performed with a resolution similar to that of $\left(e, e^{\prime} p\right)$ reactions.

\section{(d) Bound Nucleon Structure, Hadronization and Color Transparency}

These experiments will extend the domain of momentum transfers where short range effects and possibly the internal structure of the nucleons are manifested. In the Impulse Approximation, the electromagnetic response gives direct access to single nucleon densities and this description seems to provide a reasonable description for quasielastic inclusive as well as coincidence $\left(e, e^{\prime} p\right)$ results. However, when the cross sections are separated into longitudinal $(L)$ and transverse $(T)$ responses, this simple model seems to break down. The transverse response agrees reasonably well with traditional nuclear theory calculations once 
meson exchange and short range correlations are taken into account. For the longitudinal response, the models always overpredict the experimental results and this is also reflected in the longitudinal sum rule results. This is also evident in the recent ${ }^{4} \mathrm{He}\left(e, e^{\prime} p\right)$ experiment at SACLAY ${ }^{[59]}$ where an $\mathrm{L} / \mathrm{T}$ separation was performed up to $\mathrm{q}=0.7 \mathrm{GeV} / \mathrm{c}$. The $\mathrm{L} / \mathrm{T}$ ratio of the response functions shows a reduction of about $30 \%$ even after FSI and MEC are applied. When these results are combined with those of the EMC effect ${ }^{[60]}$, there is question of a possible modification of the nucleon structure within the nuclear nedium. These results are consistent with $\left(e, e^{\prime}\right)$ data from Bates ${ }^{[61]}$ at $Q^{2}$ values up to $0.4(\mathrm{GeV} / \mathrm{c})^{2}$, but are contradicted by recent inclusive $\left(e, e^{\prime}\right)$ results obtained at $S L A C^{[62]}$ at $Q^{2}=0.88$ $(\mathrm{GeV} / \mathrm{c})^{2}$, which are consistent with no longitudinal suppression. At CEBAF, Proposal [51] will extend these studies to $\mathrm{Q}^{2}=4.1(\mathrm{GeV} / \mathrm{c})^{2}$ in ${ }^{3} \mathrm{He}$ and ${ }^{4} \mathrm{He}$.
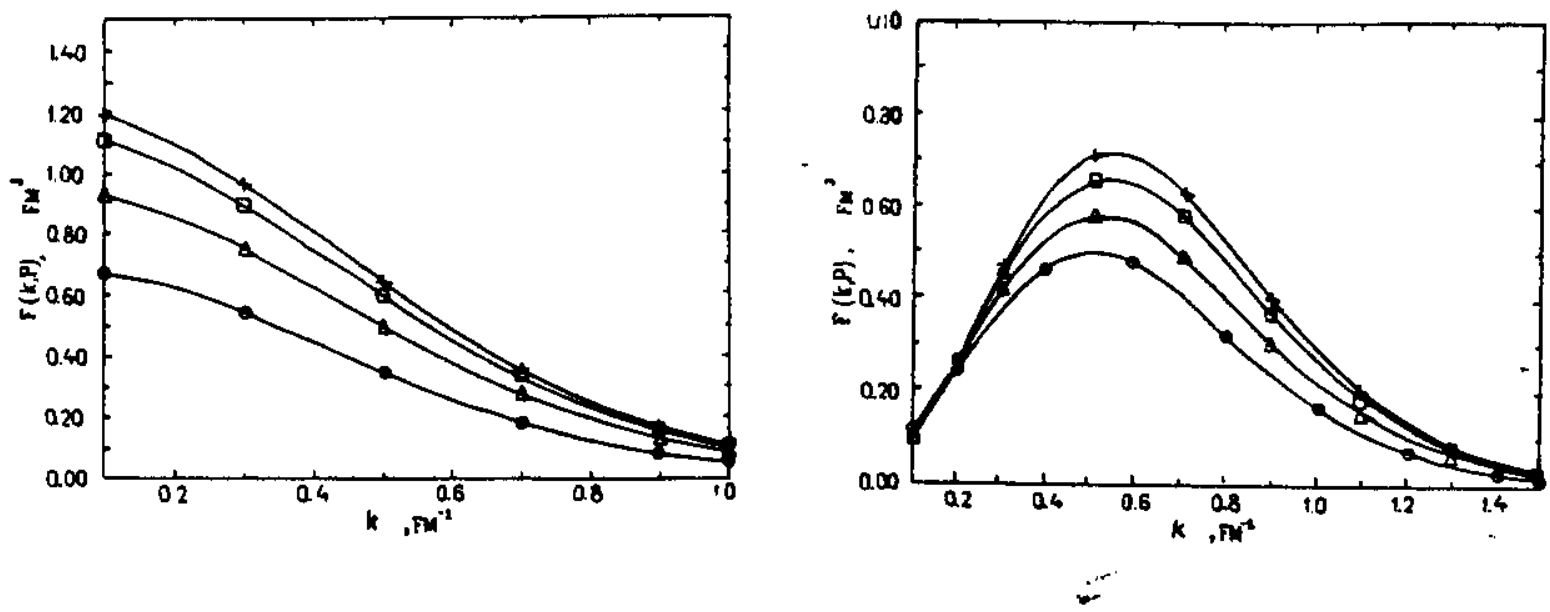

Figure 10. $12 C\left(e, e^{\prime} p\right)$ reaction for $(a)$ the $1 s_{1 / 2}$ shell and $(b)$ the $1 p_{3 / 2}$ shell. Calculations ${ }^{[88]}$ for the momentum distributions $F\left(k, p_{N}\right)$ for no transparency (standard Glauber theory): $v=0$ (circles); and for $v=1$ for $p=5 \mathrm{GeV} / \mathrm{c}$ (triangles); $p=10 \mathrm{GeV} / \mathrm{c}$ (squares) and $p=15 \mathrm{GeV} / \mathrm{c}$ (plusses).

At high $Q^{2}$, Brodsky ${ }^{[63]}$ and Muller ${ }^{[64]}$ have predicted the possible occurance of "color transparency" in nuclei. Since the amplitudes for exclusive processes involve a factor $1 / Q^{2}$ for each constituent ${ }^{[65]}$, at large momentum transfer the valence state, corresponding to the lowest number of constituents, is expected to be dominant. Since this 3-quark $\mid q q q>$ point like configuration has small color electric dipole moment it interacts only weakly with nuclear matter. If such a hadron absorbs a large momentum without additional particle production, it is contracted to a small size whose transverse spatial extent is given by $1 / Q$ from the uncertainty principle. It then evolves back to its standard configuration within a distance from the interaction point, $1_{h}$, which increases with increasing $\mathrm{Q}^{2}$. Explicit models to describe the evolution of the hadronic cross section associated with the occurance of color transparency, in the context of the parton model and PQCD have been proposed ${ }^{[66,67]}$. Experimental evidence of color transparency can also be inferred from (e,e'p) reactions at high momentum transfers by studying the $A$ and $Q^{2}$ dependence of the nuclear absorptionof the knocked out proton. Color transparency is expected to lead to a modification of the final state interaction (FSI) of the struck nucleon with respect to the prediction of the 
conventional picture in which the nucleons are assumed to be structureless. Frankfurt, Strikman and Zlialov ${ }^{[68]}$ have examined the possibility to observe color transparency in $\left(e, e^{\prime} p\right)$ reactions to specific final hole states in the residual (A-1) nucleus (see Fig 10). Several (e,e'p) experiments $\left.{ }^{[69,70}\right]$ have been proposed at CEBAF to investigate the possible onset of this effect by making cross section measurements up to $\mathrm{Q}^{2} \sim 6(\mathrm{GeV} / \mathrm{c})^{2}$ on several nuclei.

Proposal [70] also plans to measure the normal component of the recoil polarization of the ejected proton, $\mathrm{P}_{n}$. In the absence of any nuclear medium effects this polarization variable is independent of the polarization of the beam and should be zero to first order in PWIA due to time reversal symmetry. Hence this effect can be used to great advantage as an effective filter in the study of FSI in nuclei and also to observe the onset of color transparency in nuclei at high momentum transfers.

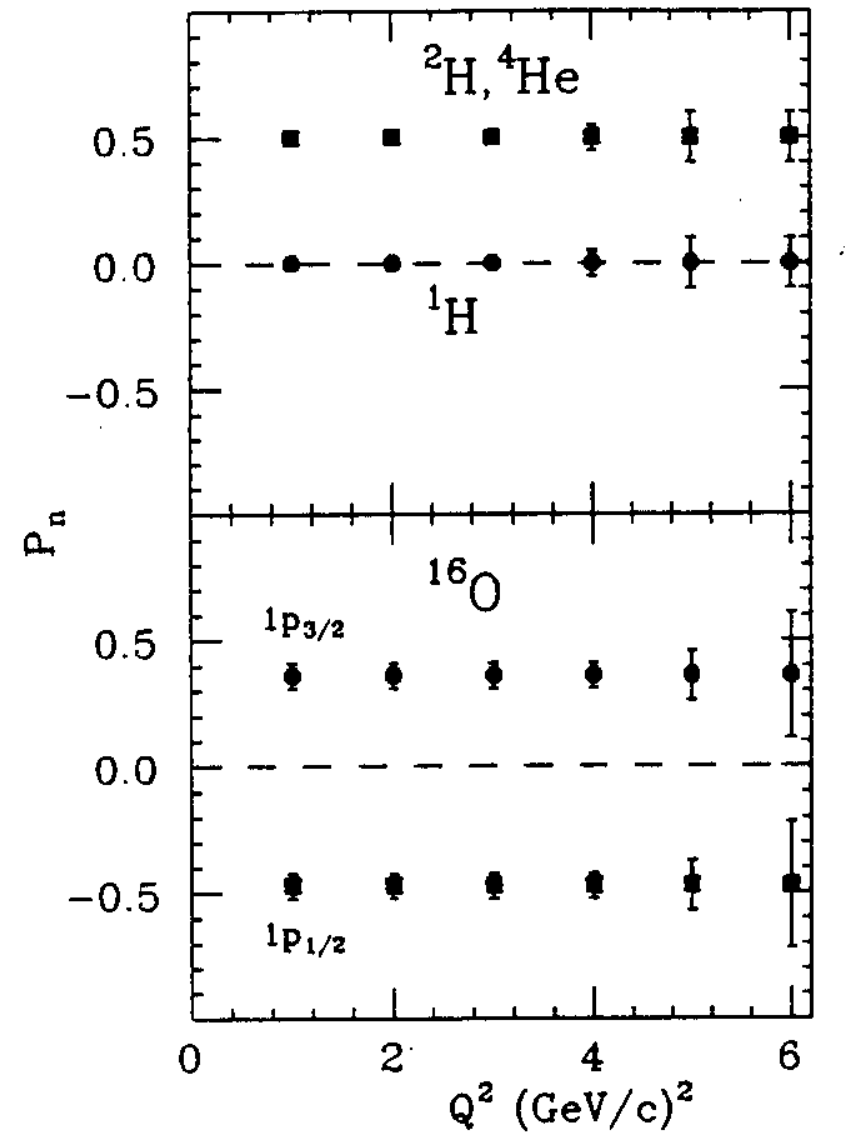

Figure 11. Projected absolute uncertainties in $P_{n}$ from the proposed $\left(e, e^{\prime} \overrightarrow{\mathrm{p}}\right)$ experiment ${ }^{[70]}$ on the light nuclei $\left({ }^{1} \mathrm{H},{ }^{2} \mathrm{H}\right.$ and $\left.{ }^{4} \mathrm{He}\right)$ and ${ }^{16} \mathrm{O}$ permit distinguishing between various models. For ${ }^{1} \mathrm{H}, P_{n}=0$ for all $Q^{2}$ values, whereas for ${ }^{2} H$ and ${ }^{4} \mathrm{He}$ the $P_{\mathrm{n}}=0.5$ value was cho. sen for illustrative purposes only to show the absolute uncetainty with which we are able to determine $P_{n}$. Calculations [71] for $P_{n}$ for ${ }^{16} \mathrm{O}$ at $Q^{2} \sim 1.0(\mathrm{GeV} / \mathrm{C})^{2}$ for the $p_{3 / 2}$ level is $\sim 0.36$ and for the $p_{1 / 2}$ level is $\sim .0 .47$.

\section{SUMMARY}

We have presented here most of the few-body physics programs which have been submitted to the CEBAF Program Advisory Comnittee to be performed in Hall A. High resolution (200-500 keV missing mass resolutions and $\sim 1 \mathrm{MeV}$ for few nucleon systems) and high accuracy (absolute cross section to $\sim 1 \%$ accuracy) coincidence experiments in the few $\mathrm{GeV}$ region will be the primary goal of Hall $\mathrm{A}$. In essence, it will fully match the unique characteristics of the CEBAF beam $-4 \mathrm{GeV}$ incident energy, $100 \%$ duty factor, $200 \mu \mathrm{A}$ beam intensity and a momentum resolution of $10^{-4}$ - to perform exclusive as well as inclusive electromagnetic measurements with both high precision and accuracy. 


\section{REFERENCES}

[1] H.A. Grunder, $4^{\text {th }}$ Wkshp on Perspectives in Nuclear Physics at Intermediate Energies, ICTP, Italy, May 8-12, 1989, S. Boffi et al., eds., World Scientific, Singapore (1989).

[2] L.S. Cardman et al., Univ. of Illinois Technical Note TN-89-4 (1989).

[3] Conceptual Design Report, Basic Experimental Equipment, CEBAF (1990).

(4) D.G. Crabbe et al., Phys. Rev. Lett 64, 2627 (1990).

5. R. Madey et al., IEEE Transactions on Nuclear Science 36, 231 (1989).

[6] A. Zondervan et al., Topical Wkshp on Two-Nucleon Emission Reactions, EIPC, Elba, Italy, Sept. 19-23 (1989).

[7] S. Frullani et al., "Multi Purpose Spectrmeter", INFN-ISS 90/5 (1990).

(8) S. Platchkov et al., Nucl. Phys. A510, 740 (1990).

[9] R.G. Arnold, Spokesperson, SLAC Proposal NE11; P.E. Bosted et al., SLAC-PUB5744 (1992).

[10] N. Dombey, Rev. Mod. Phys 41, 236 (1969).

[11] R.G. Arnold, C.E. Carlson and F. Gross, Phys. Rev. 23, 363 (1981).

[12] CEBAF Proposal 89-018, D. Day, spokesperson (1989).

13] CEBAF Proposal 89-005, R. Madey, spokesperson (1989).

14] CEBAF Proposal 89-014, C.F. Perdrisat and V. Punjabi, spokespersons (1989).

15] CEBAF Proposals 89-028, 89-026, J.M. Finn and P.E. Ulmer, spokespersons (1989).

[16] H. Ahrenhovel, Priv. Comm.. See also References 40,41.

17] S. Weinberg, Phys. Rev. D5, 1412 (1972)

[18] J. Ashman et al., Nucl. Phys. B328, 1 (1989).

[19] L.A. Ahrens (et al., Phys. Rev. D35, 785 (1987).

[20] J. Napolitano, Phys. Rev C43, 1473 (1991).

(21] R.L. Jaffe, Phys. Lett. B229, 275 (1989).

[22] N.W. Park et al., Phys. Rev. D43, 869 (1991).

[23] CEBAF Proposal 91-010, P. Souder and J.M. Finn, spokespersons (1991).

[24] CEBAF Proposal 91-004, E. Beise, spokesperson (1991) :-

[25] CEBAF Proposal 91-017, D. Beck, spokesperson (1991).

[26] MIT Bates "SAMPLE" collaboration, D. Beck and R. McKeown, spokespersons (1989).

[27] V. Burkert, "Electroproduction of Light Quark baryons", CEBAF-PR-92-01 (1992).

[28] CEBAF Proposal 91-011, R. Lourie and S. Frullani, spokespersons (1991).

[29] CEBAF Proposal 91-026, G. Petratos, spokesperson (1991).

[30] S. Auffret et al., Phys. Rev. Lett 55, 1362 (1985).

[31] R.G. Arnold et al., Phys. Rev. C42, R1 (1990).

[32] CEBAF Proposal 89-047, J. Jourdan, G.G. Petratos, J. Mougey, spokespersons (1989).

33] J. Napolitano et al., Phys. Rev. Lett. 61, 2530 (1988).

[34] CEBAF Proposal 89-019, R. Holt, R. Gilman, Z.E. Meziani, spokespersons (1989).

[35] T. Kamae et al., Nucl. Phys. B139, 394 (1978); Phys. Rev. Lett 38, 468 (1977); H. Ikeda et al., Nucl. Phys. B172, 509 (1980); Phys. Rev. Lett 42, 1321 (1979).

[36] F.F. Liu et al., Phys. Rev. 165, 1478 (1968).

[37] A.S. Bratashevskii it et al., Yad. Fiz. 31, 860 (1980) [Sov. J. Nucl. Phys. 31, 444 (1980)]; JETP Lett. 34, 389 (1982); JETP Lett. 36, 216 (1983).

[38] T. Kamae and T. Fujita, Phys. Rev. Lett. 38, 471 (1977).

[39] J.M. Laget, Nucl. Phys. A312, 265 (1978).

[40] W. Fabian and H. Arenhovel, Nucl. Phys. A314, 253 (1979).

[41] A.Yu. Korchin et al., Sov. J. Nucl. Phys. 48, 243 (1988).

[42] M.P. Rekalo et al., J. Phys. G15, 1223 (1989) 
[43] F. Gross and J.W. Van Orden, private communication.

[44] R.G. Arnold et al., Phys. Rev. Lett. 40, 1429 (1978).

[45] J.M. Cavedon et al., Phys. Rev. Lett. 49, 987 (1982).

[46] F-P. Juster et al., Phys. Rev. Lett. 55, 2261 (1985).

[47] CEBAF Proposal 89-021, G. Petratos, spokesperson (1989).

[48] M. Bernheim et al., Nucl. Phys. A365, 349 (1981).

[49] S. Turck-Chieze et al., Phys. Lett. 142B, 145 (1984).

[50] C. Marchand et al., Phys. Rev. Lett. 60, 1703 (1988)

[51] CEBAF Proposal 89-044, J. Mougey, A. Saha, M. Epstein and R. Lourie, spokespersons (1989).

[52] C. Ciofi degli Atti et al., Phys. Lett. A449, 219 (1986).

[53] J.M. Legoff, $4^{\text {th }}$ Wkshp on Perspectives in Nuclear Physics at Intermediate Energies, ICTP, Italy, May 8-12, 1989, S. Boffi et al., eds., World Scientific, Singapore (1989).

[54] P. E. Ulmer et al., Phys. Rev. Lett. 59, 2259 (1987).

[55] R. W. Lourie et al., Phys. Rev. Lett. 56, 2364 (1986).

[56] H. Baghaei et al., Phys. Rev. C39, 177 (1989).

[57] CEBAF Proposal 89-030, R.A. Lindgren, M.Epstein, G. Lolos and Z. Meziani, spokespersons (1989).

[58] CEBAF Proposal 89-029, H.P. Blok, spokesperson (1989).

[59] A. Magnon et al., Phys. Lett. 222B, 352 (1989) and priv. comm..

[60] J.J. Aubert et al., Phys. Lett. 123B, 275 (1983).

61] K.F. von Redden et al., Phys. Rev. C41, 1084 (1990).

62] Z.-E. Meziani et al., Phys. Rev. Lett., to be published (1992).

[63] S.J. Brodsky, in Proceedings of the Thirteenth International Symposium on Multipar. ticle Dynamics. Eds. E.W. Kittel, W. Metzger and A. Stergion (World Scientific, Singapore), 963 (1982).

[64] A. Muller, in Proceedings of the Seventh Recontre de Moriond. Ed. J. Thran Thanh Van (Editions Frontieres, Gif-sur-Yvette), 13 (1982).

[65] S.J. Brodsky and G.R. Farrar, Phys. Rev. Lett. 31, 1153 (1973).

[66] G.R. Farrar et al, Phys. Rev. Lett. 62, 1095 (1989).

[67] O. Benhar, priv. comm..(1991).

[68] L.L. Frankfurt, M.I. Strikman and M.B. Zhalov, Nucl. Phys. A515, 599 (1990) and M.B. Zhalov, priv. comm. (1990).

[69] CEBAF Proposal 91-007, R. Milner, spokesperson (1991); CEBAF Proposal 91-013, D. Geesaman, spokesperson (1991)

[70] CEBAF Proposal 91-006, A. Saha, spokesperson (1991)

[71] A. Picklesimer et al, Phys. Rev. C32, 1312 (1985); A. Picklesimer and J.W. van Orden, Phys. Rev. C40, 290 (1989); J.W. van Orden, priv. comm. (1991). 